\title{
PRÉ-NATAL DO PARCEIRO NA ATENÇÃO PRIMÁRIA À SAÚDE
}

\author{
PRENATAL PARTNER IN PRIMARY HEALTH CARE
}

\author{
Pamela Reis Martins ${ }^{1}$ \\ Luciana Modesto de Brito $^{2}$ \\ Cícera Amanda Mota Seabra ${ }^{3}$ \\ Ankilma do Nascimento Andrade Feitosa ${ }^{4}$
}

RESUMO: Introdução: As mudanças fisiológicas que a gestação traz são vivenciadas pela mulher, isto faz com que a paternidade, culturalmente, só seja encarada a partir do nascimento da criança. Por isso, as políticas de saúde têm incluído cada vez mais o pai durante as consultas do Pré-natal, de forma a buscar estreitar laços familiares, criar vínculos e até cuidar da saúde do homem. O Pré-natal do parceiro na Atenção Primária à Saúde surge como uma oportunidade para dar informações ao casal acerca das mudanças naturais do corpo, do parto e do que

\footnotetext{
${ }_{1}$ Médica. Residente em Saúde da Família e Comunidade da Faculdade Santa Maria, Cajazeiras, Paraíba.

2 Mestra em Sistemas Agroindustriais pela UFCG. Pós-graduanda em Cardiologia. Graduada em Medicina pela Faculdade de Medicina Nova Esperança FAMENE (2011). Especialização em Preceptoria de Residência Médica no SUS pelo Hospital Sírio Libanês. Especialização em Medicina Intensiva pela Faculdade Redentor, Rio de Janeiro. Atualmente Médica Intervencionista do Serviço de Atendimento Móvel de Urgência (SAMU), Médica diarista do Hospital Padre Alfredo Barbosa), atua na Faculdade Santa Maria como Docente do curso de medicina, coordenadora do Internato Médico e responsável técnica da Clínica Santa Maria, Cajazeiras, Paraíba.

3 Médica com graduação em Medicina pela Universidade Federal de Campina Grande (2008). Trabalhou por 8 meses como médica contratada do Programa de Saúde da Família da Prefeitura Municipal de Itapipoca de maio a dezembro de 2008. Concluiu Residência Médica pelo Programa de Pós-Graduação em Residência de Medicina de Família e Comunidade do Sistema Municipal de Saúde Escola da Prefeitura Municipal de Fortaleza com duração de 2 anos, tendo iniciado em janeiro de 2009 e concluído em janeiro de 2011. Possui Título de Especialista em Medicina de Família e Comunidade pela Associação Médica Brasileira. Exerceu a função de Médica de Família e Comunidade concursada do Programa de Saúde da Família da Prefeitura Municipal de Aurora-CE desde janeiro de 2011 a março de 2019, atualmente é médica concursada da Prefeitura Municipal de Barbalha-CE e, também, é supervisora do Programa Mais Médicos para o Brasil desde janeiro de 2014. Desde fevereiro de 2016 exerce a função de professora contratada da Faculdade Santa Maria (FSM) em Cajazeiras - PB das disciplinas Programa de Aprendizagem da Atenção Básica (PAAB) IV e VIII, Semiologia e Saúde do Idoso, e está na coordenação do Programa de Residência de Medicina de Família e Comunidade em Cajazeiras-PB.

${ }^{4}$ Pós-doutorado pela Universidade Federal de Campina Grande. Doutora em Ciências da Saúde pela Faculdade de Medicina do ABC. Docente da Faculdade Santa Maria, Cajazeiras, Paraíba.
} 
esperar durante o puerpério, por exemplo, assim como realizar testes e exames no parceiro com objetivo de identificar doenças que devem ser tratadas pelo casal, como a sífilis. Objetivo: Identificar a importância do envolvimento do homem no prénatal como forma de quebrar as barreiras da construção social de gênero. Método: Estudo bibliográfico com abordagem qualitativa realizada durante o período de setembro de 2021 a janeiro de 2022 pelas bases de dados Literatura e National Library of Medicine (PUB-MED), Biblioteca Nacional em Saúde (BVS) e Scientific Electronic Library Online (SciELO). Como critérios de inclusão foram utilizados artigos nacionais e internacionais que estivessem disponíveis nas bases de dados citadas, com resumo disponíveis e artigo de disponibilização gratuita publicados entre o ano de 2016 e 2021. Resultados: Inúmeros benefícios são alcançados quando o companheiro se envolve nas consultas de pré-natal, como a formação de vínculo pai-mãe-bebê, oportunidade para cuidar da saúde do homem com a realização de exames e rastreamento de doenças, momento de obter informações para todo o ciclo gravídico-puerperal de forma a incentivar a participação ativa durante o parto, além de favorecer a prevalência do aleitamento materno, já que a mulher terá um suporte ainda maior, principalmente no compartilhamento de atividades domésticas, dentre outros benefícios. Como barreira para esta participação está a dificuldade de conciliar os horários de abertura dos serviços de saúde com suas atividades laborais. Para isso, os profissionais de saúde da Estratégia Saúde da Família devem buscar estratégias para diminuir essas barreiras e incluir a participação masculina nas consultas e outras atividades realizadas. Conclusão: Portanto, conclui-se que apesar da participação ser mínima, ela ainda deve ser incentivada para garantir uma vivência exitosa da mulher e de toda a sua família nessa fase importante da vida, tendo em vista que existem políticas que respaldam a participação do companheiro no pré-natal e os benefícios são cientificamente comprovados.

Palavras-chave: Pré-Natal, Atenção Primária à Saúde, Paternidade.

ABSTRACT: Introduction: The physiological changes that pregnancy brings are experienced by the woman, this makes fatherhood, culturally, only seen from the birth of the child. Therefore, health policies have increasingly included the father during prenatal consultations, in order to seek to strengthen family ties, create bonds and even take care of men's health. The prenatal care of the partner in the Primary Health Care emerges as an opportunity to give information to the couple about the natural changes of the body, childbirth and what to expect during the puerperium, for example, as well as to perform tests and tests on the partner in order to identify diseases that should be treated by the couple, such as syphilis. Objective: To identify the importance of male involvement in prenatal care as a way to break down the barriers of social construction of gender. Method: Bibliographic study with qualitative approach carried out during the period from September 2021 to January 2022 by the databases Literatura e National Library of Medicine (PUB-MED), Biblioteca Nacional em Saúde (VHL) and Scientific Electronic Library Online (SciELO). National and international articles that were available in the cited databases were used as inclusion criteria, with available summary and free available article published between 2016 and 2021. Results: Numerous benefits are achieved 
when the partner is involved in prenatal consultations, such as the formation of a father-mother-baby bond, opportunity to take care of the health of the man with the performance of tests and screening of diseases, time to obtain information for the entire pregnancy-puerperal cycle in order to encourage active participation during childbirth, in addition to favoring the prevalence of breastfeeding, since women will have even greater support, especially in the sharing of domestic activities, among other benefits. As a barrier to this participation is the difficulty of reconciling the opening hours of health services with their work activities. For this, health professionals of the Family Health Strategy should seek strategies to reduce these barriers and include male participation in consultations and other activities performed. Conclusion: Therefore, it is concluded that although participation is minimal, it should still be encouraged to ensure a successful experience of women and their entire families in this important phase of life, considering that there are policies that support the participation of the partner in prenatal care and the benefits are scientifically proven.

Keywords: Prenatal. Primary Health Care. Paternity. 


\section{INTRODUÇÃO}

O período gestacional é marcado por diversas transformações na vida da mulher. Neste período, é o momento de procurar ajuda especializada para realização do pré-natal e preparação do parto, buscar informações e realizar exames periódicos para sua saúde e a do bebê, além disso, é fundamental que a mulher conte com apoio da família e do seu companheiro (BALICA; AGUIAR, 2019).

$\mathrm{Na}$ maioria dos casos, o foco maior do pré-natal é a gestante, porém os serviços de saúde buscam cada vez mais a inclusão do homem/pai durante todo o acompanhamento, seja nas consultas ou nas rodas de conversas e palestras realizadas pela equipe. É sabido que culturalmente a paternidade só se inicia com o nascimento do seu filho, mas essa realidade tem se modificado através dos estudos sobre o papel do pai na gestação (SANTOS; KREUTZ, 2014).

Por isso, em 2016 o Ministério da Saúde criou uma estratégia com objetivo de envolver os homens de forma consciente e ativa em todas as áreas do planejamento reprodutivo de forma a contribuir para maior procura e acesso desse público aos serviços de saúde que ainda é reduzida, com enfoque principalmente na Atenção Básica de Saúde, que é o Pré-Natal do Parceiro (BRASIL, 2018).

Esse incentivo vem diante do contexto histórico em que o planejamento reprodutivo e as ações voltadas para o momento da gestação, parto e puerpério são pensadas e idealizadas com foco na mulher e no binômio mãe-filho. No entanto, esse cenário tem se modificado nos últimos anos não só no Brasil, mas em todo o mundo em que se é discutida a importância do parceiro em todas as fases do planejamento e gestação (BRASIL, 2018; MENDES; SANTOS, 2019).

A política de integralidade do Sistema Único de Saúde (SUS) é incentivada quando, aos poucos, o pai é trazido para o contexto gestacional e compreende, junto com a mulher, as mudanças naturais do gestar, pois a identidade paterna leva um tempo para se desenvolver tendo em vista que as modificações no corpo quem vivencia é a mulher. No entanto, o companheiro pode participar ativamente das 
consultas de pré-natal para compreendê-las (FERREIRA et al., 2016; BALICA; AGUIAR, 2019).

Por fim, a partir do momento que o homem comparece à Atenção Básica de Saúde se torna um momento oportuno para cuidar da sua saúde, realizar testes rápidos, consultas com os profissionais e verificar se possui alguma doença, pois o tratamento da sífilis, por exemplo, se detectado, a gestante só é curada completamente quando o parceiro também for tratado. Em casos em que o tratamento não é realizado no companheiro, a contaminação persiste e pode trazer consequências para o bebê (BALICA; AGUIAR, 2019).

Neste sentido, diante das práticas de pré-natal na Unidade de Estratégia em Saúde da Família em que pude vivenciar a presença nas consultas e educação em saúde apenas da presença feminina surgiu o interesse em trabalhar a temática e contribuir para a comunidade acadêmica e sociedade da importância da presença ativa do homem durante todas as fases da pré-concepção, gestação e puerpério.

Neste sentido, este estudo se torna relevante por ampliar o conhecimento acerca de quais estratégias podem ser implementadas pela Unidade Básica de Saúde para trazer o companheiro para dentro do serviço que pode ser uma porta de entrada para oferta dos demais cuidados com relação à saúde do homem.

Visto isso, o objetivo do estudo é identificar a importância do envolvimento do homem no pré-natal como forma de quebrar as barreiras da construção social de gênero, bem como investigar quais fatores influenciam a participação e não participação durante as consultas e apresentar estratégias de adesão que podem ser adotadas pelas Unidades de Saúde da Família para participação do homem no pré-natal.

\section{MÉTODO}

Trata-se de uma revisão da literatura com abordagem qualitativa realizada no período compreendido entre setembro de 2021 a janeiro de 2022 com objetivo de 
identificar a importância do envolvimento do homem no pré-natal de forma ativa como estratégia de quebrar as barreiras da construção social de gênero.

A revisão da literatura é uma análise bibliográfica mais detalhada a partir de estudos já publicados de um determinado assunto, envolve localizar, explorar, resumir e interpretar a investigação prévia com uma área do assunto (BENTO, 2012).

Para busca de artigos foram utilizadas as bases de dados Literatura e National Library of Medicine (PUB-MED), Biblioteca Nacional em Saúde (BVS) e Scientific Electronic Library Online (SciELO).

Os Descritores em Ciências da Saúde (DeCS) utilizados foram Pré-Natal, Atenção Primária à Saúde e Paternidade. Os critérios de inclusão contemplados foram artigos nacionais e internacionais que estivessem disponíveis nas bases de dados citadas, com resumo disponíveis e artigo de disponibilização gratuita publicados entre o ano de 2016 e 2021. Os critérios de exclusão definidos foram artigos incompletos, teses e dissertações que não possuíam relação direta com a temática abordada.

Após estabelecidos os critérios, iniciou-se a coleta de dados que consiste na aplicação dos descritores e critérios de inclusão e exclusão nas bases de dados para selecionar e resumir um conjunto de dados que podem ser facilmente utilizados após seleção para compor a pesquisa.

Foram escolhidos os descritores e seu operador booleano: "Pré-Natal and Paternidade", "Pré-Natal and Atenção Primária à Saúde and Paternidade".

Após a coleta, os dados foram tabulados com o auxílio do Microsoft Excel 2010 para descrever os estudos selecionados e quais os principais objetivos e achados encontrados pelos autores (Tabela 1) e discutidos logo adiante.

\section{RESULTADOS E DISCUSSÃO}

Após cruzamento dos critérios de inclusão e descritores foram encontrados 01 artigo na BVS, 02 na Scielo e 125 na PubMed. Foram selecionados 18 artigos após 
leitura do título e resumo superficialmente para estudo aprofundado do conteúdo. Após esta etapa, 07 artigos foram inclusos na pesquisa por satisfazer os critérios de inclusão estabelecidos. Na Tabela 1 estão descritos os artigos selecionados para compor a pesquisa e os objetivos e conclusão obtidos.

Tabela 1 - Artigos selecionados para compor o estudo.

\begin{tabular}{|c|c|c|c|c|c|}
\hline TÍTULO & AUTORES & ANO & $\begin{array}{l}\text { BASE DE } \\
\text { DADOS }\end{array}$ & $\begin{array}{c}\text { OBJETIVOS DA } \\
\text { PESQUISA }\end{array}$ & CONCLUSÃO \\
\hline $\begin{array}{l}\text { A visão das } \\
\text { gestantes } \\
\text { acerca da } \\
\text { participação } \\
\text { do homem } \\
\text { no processo } \\
\text { gestacional. }\end{array}$ & $\begin{array}{l}\text { CALDEIRA, } \\
\text { Letícia } \\
\text { Ábdon et al. }\end{array}$ & 2017 & BVS & 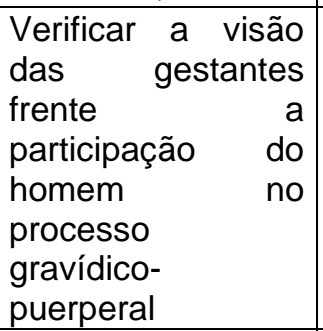 & $\begin{array}{l}\text { Evidenciou a importância da } \\
\text { participação do } \\
\text { companheiro diante do } \\
\text { benefício da formação de } \\
\text { vínculo entre pai-mãe-filho e } \\
\text { de como a Atenção Primária } \\
\text { à Saúde pode contribuir } \\
\text { com esse processo. }\end{array}$ \\
\hline $\begin{array}{l}\text { Participação } \\
\text { do } \\
\text { companheiro } \\
\text { da gestante } \\
\text { nas } \\
\text { consultas de } \\
\text { pré-natal: } \\
\text { prevalência e } \\
\text { fatores } \\
\text { associados. }\end{array}$ & $\begin{array}{l}\text { BRITO, } \\
\text { Jaqueline } \\
\text { Guimarães } \\
\text { Elói, et al. } \\
\end{array}$ & 2021 & SCIELO & $\begin{array}{l}\text { Identificar os } \\
\text { fatores associados } \\
\text { a participação do } \\
\text { companheiro nas } \\
\text { consultas de pré- } \\
\text { natal }\end{array}$ & $\begin{array}{l}\text { Participação } \\
\text { companheiros nas consultas } \\
\text { maior em casais que } \\
\text { planejaram a gravidez, } \\
\text { mulheres com alta } \\
\text { escolaridade, } \\
\text { realizaram seis ou mais } \\
\text { consultas de pré-natal e em } \\
\text { serviços privados. }\end{array}$ \\
\hline $\begin{array}{l}\text { Adaptação } \\
\text { de } \\
\text { mensagens } \\
\text { educativas } \\
\text { para } \\
\text { parceiros de } \\
\text { gestantes } \\
\text { para uso em } \\
\text { tecnologias } \\
\text { móveis em } \\
\text { saúde } \\
\text { (mHealth). }\end{array}$ & $\begin{array}{l}\text { BONIFÁCIO, } \\
\text { Lívia } \\
\text { Pimenta; } \\
\text { SOUZA, } \\
\text { João Paulo; } \\
\text { VIEIRA, } \\
\text { Elisabeth } \\
\text { Meloni. }\end{array}$ & 2019 & SCIELO & $\begin{array}{l}\text { Desenvolvimento } \\
\text { de mensagens de } \\
\text { textos r para } \\
\text { parceiros de } \\
\text { mulheres grávidas. }\end{array}$ & $\begin{array}{l}\text { Foram realizados testes e } \\
\text { construído mensagens de } \\
\text { texto que incentivavam a } \\
\text { busca por informação no } \\
\text { contexto gestacional. }\end{array}$ \\
\hline $\begin{array}{l}\text { Father's } \\
\text { participation } \\
\text { in prenatal } \\
\text { care and } \\
\text { childbirth: } \\
\text { contributions } \\
\text { of nurses' } \\
\text { interventions. }\end{array}$ & $\begin{array}{l}\text { LIMA, } \\
\text { Katherine } \\
\text { Souza Vidal, } \\
\text { et al. }\end{array}$ & 2021 & PUBMED & $\begin{array}{lr}\text { Descrever } & 0 \\
\text { discurso } & \text { dos } \\
\text { homens/pai } & \text { sobre } \\
\text { a participação } & \text { no } \\
\text { pré-natal } & \text { e parto } \\
\text { dos seus } & \text { fillos a } \\
\text { partir da ajuda de } \\
\text { enfermeiras. }\end{array}$ & $\begin{array}{l}\text { Evidenciou que o processo } \\
\text { de participação paterna } \\
\text { nesse contexto gravídico- } \\
\text { puerperal está em } \\
\text { construção e que o incentivo } \\
\text { de profissionais possibilitou } \\
\text { uma maior adesão e de } \\
\text { ressignificação } \\
\text { identidade masculina diante } \\
\text { da gestação e do parto. }\end{array}$ \\
\hline
\end{tabular}




\begin{tabular}{|c|c|c|c|c|c|}
\hline $\begin{array}{l}\text { Paternal } \\
\text { Involvement } \\
\text { and Maternal } \\
\text { Perinatal } \\
\text { Behaviors: } \\
\text { Pregnancy } \\
\text { Risk } \\
\text { Assessment } \\
\text { Monitoring } \\
\text { System, } \\
\text { 2012-2015 }\end{array}$ & $\begin{array}{l}\text { KORTSMIT, } \\
\text { Katherine et } \\
\text { al. }\end{array}$ & 2020 & PUBMED & $\begin{array}{l}\text { Comparar a } \\
\text { participação } \\
\text { paterna diante de } \\
\text { mulheres casadas, } \\
\text { solteiras, mas com } \\
\text { reconhecimento } \\
\text { paterno ou } \\
\text { solteiras sem } \\
\text { reconhecimento } \\
\text { paterno. }\end{array}$ & $\begin{array}{l}\text { Os resultados trazem que } \\
\text { as mulheres casadas, com } \\
\text { apoio do homem, têm mais } \\
\text { propensão de iniciar o } \\
\text { acompanhamento do pré- } \\
\text { natal precocemente, assim } \\
\text { como continuar com o } \\
\text { aleitamento materno. }\end{array}$ \\
\hline $\begin{array}{l}\text { Experiences, } \\
\text { views and } \\
\text { needs of } \\
\text { first-time } \\
\text { fathers in } \\
\text { pregnancy- } \\
\text { related care: } \\
\text { a qualitative } \\
\text { study in } \\
\text { south-east } \\
\text { Nigeria }\end{array}$ & $\begin{array}{l} \\
\text { ONYEZE- } \\
\text { JOE, } \\
\text { Chiemeka; } \\
\text { GODIN, } \\
\text { Isabelle. } \\
\end{array}$ & 2020 & PUBMED & $\begin{array}{lr}\text { ldentificar } & \text { a } \\
\text { experiência } & \text { de } \\
\text { pais de primeira } \\
\text { viagem } \\
\text { envolvimento no na } \\
\text { gestação. }\end{array}$ & $\begin{array}{l}\text { Identificou que, apesar da } \\
\text { inexperiência, a rede de } \\
\text { apoio e o acesso a } \\
\text { informação contribuiu para } \\
\text { uma experiencia exitosa. } \\
\text { Assim como, encontrou que } \\
\text { as atividades laborais } \\
\text { impactam diretamente na } \\
\text { não participação ativa dos } \\
\text { companheiros. }\end{array}$ \\
\hline $\begin{array}{l}\text { Barriers to } \\
\text { men's } \\
\text { participation } \\
\text { in perinatal } \\
\text { care: a } \\
\text { qualitative } \\
\text { study in Iran }\end{array}$ & $\begin{array}{l}\text { FIROUZAN, } \\
\text { Vahideh et } \\
\text { al. }\end{array}$ & 2019 & PUBMED & $\begin{array}{lr}\text { Determinar } & \text { as } \\
\text { barreiras } & \text { que } \\
\text { dificultam } & \text { a } \\
\text { participação } & \text { do } \\
\text { homem } & \text { na } \\
\text { gestação. } & \end{array}$ & $\begin{array}{l}\text { Foram encontrados quatro } \\
\text { aspectos que contribuem } \\
\text { para essa não participação: } \\
\text { as barreiras culturais, as } \\
\text { barreiras pessoais, as } \\
\text { barreiras relacionadas ao } \\
\text { serviço de saúde e as } \\
\text { barreiras socioeconômicas. }\end{array}$ \\
\hline
\end{tabular}

Fonte: Próprio Autor, 2022.

Apesar da gestação, parto e pós-parto serem considerados uma luta essencialmente feminina, a luta pela humanização trouxe um olhar diferenciado para a participação masculina neste cenário, evidenciando benefícios comprovados no exercício da paternidade ativa durante todo o ciclo gravídico-puerperal (LIMA et al., 2021).

O pré-natal é a estratégia utilizada para garantir o acompanhamento durante a gestação e garantir a saúde materna e neonatal. Durante as consultas a gestante pode tirar dúvidas, expor medos e inseguranças, recebendo as orientações necessárias para ser a protagonista do seu parto (COSTA et al., 2016; GOMES; FILHA; PORTELA, 2017).

Neste sentido, a presença paterna durante este acompanhamento é essencial para prepará-lo também para o momento, incentivando o companheirismo, 
compartilhamento da tomada de decisões e garantir a paternidade ainda na gestação (FRANCISCO et al., 2015; MAAS, 2012).

A diferença de gênero representada pela divisão de papéis na sociedade alimentada pelo patriarcado é nítida, em que há uma distinção nas funções paternas e maternas. Antigamente, à mulher cabia o papel de cuidar do lar e das crianças, além de educá-las para que o pai fosse o provedor da família, a autoridade, sem se envolver com os cuidados dos filhos (LIMA et al., 2021).

Seguindo esse pensamento, culturalmente as ações em saúde realizadas no período gravídico-puerperal são dedicadas exclusivamente à mulher e ao recémnascido, com diferenças na promoção da saúde para este binômio e para a saúde do homem. Além disso, há um entrave que perpassa décadas do não comparecimento de forma assídua do homem nos serviços de saúde, em especial na Atenção Primária (BRASIL, 2018; MOREIRA; GOMES; RIBEIRO, 2016).

Como forma de incentivo, a Política Nacional de Atenção Integral à Saúde do Homem (PNAISH) defende a participação ativa dos pais no ciclo gravídico-puerperal, buscando modificar o paradigma do binômio mãe-bebê para pai-mãe-bebê (BRASIL, 2021).

Durante o período gestacional, a mulher se encontra vulnerável e necessita do apoio familiar e um ambiente social agradável, pois isto também contribui positivamente para o desenvolvimento saudável da criança. Autores sugerem que a participação do parceiro como suporte neste período traz inúmeros benefícios (BONIFÁCIO; SOUZA; VIEIRA, 2019).

O Ministério da Saúde publicou em 2018 um Guia do Pré-Natal do Parceiro para Profissionais da Saúde que elenca o que pode ser alcançado com a presença do companheiro nas consultas: 1º realização de testes rápidos e exames de rotina no parceiro (tipagem sanguínea e fator $\mathrm{RH}, \mathrm{HBsAg}$, teste treponêmico e/ou não treponêmico para detecção de sífilis, pesquisa de anticorpos anti-HIV e anti-HCV, aferição de pressão arterial, cálculo de IMC; $2^{\circ}$ atualização do cartão de vacina do parceiro; 3ํabordagens voltadas à saúde do homem; 4ำimportância do papel do homem na gestação, parto, pós-parto e cuidados com a criança (BRASIL, 2018).

Corroborando com os objetivos do Guia do Pré-Natal do Parceiro, Lima e colaboradores (2021) trazem discursos de pais que participaram das consultas com 
as gestantes em que relatam aproveitar a oportunidade para acessar os serviços de saúde para realização de exames laboratoriais e atualização do cartão de vacina.

Estudo realizado por Caldeira e colaboradores (2017) com gestantes demonstra que o apoio oferecido a elas neste período por seus companheiros passa segurança, força, alegria, dentre outros sentimentos. Além disso, quando envolvidos com a gestação, contribuem até com o vínculo entre mãe-bebê, fazendo com que alterações durante a gravidez sejam vivenciadas de forma mais leve.

Pesquisas internacionais destacam que o envolvimento dos companheiros nos serviços de saúde materna vai além do estímulo para formação de vínculo entre pai-bebê, favorece o empoderamento masculino para conhecer as etapas do parto e passar mais confiança para os familiares de que tem uma pessoa conhecida com a mulher na maternidade (CHIKALIPO; CHIRWA; MUULA, 2018).

Segundo autores, a maioria dos companheiros não conhecem a Lei do Acompanhante (Lei no 11.108, de 07 de abril de 2005), por exemplo, assim como que podem participar de forma ativa no trabalho de parto. Neste sentido, a presença nas consultas de pré-natal amplia esse leque de informações e contribui na reivindicação dos direitos da parturiente (LIMA et al., 2021).

As gestantes relatam que a participação do companheiro as deixa felizes, com sentimento de segurança. Essa presença, por alguns autores, se iguala aos métodos não farmacológicos da dor utilizados durante o trabalho de parto, pois minimiza a ansiedade. Sendo assim, a sua participação é benéfica não só durante o pré-natal, mas também proporciona uma paternagem corajosa (CALDEIRA et al., 2017).

Devido ao apoio oferecido pelo companheiro na gestação da sua esposa, há evidências que comparam a prevalência do aleitamento materno em mães casadas com apoio e mães solteiras sem apoio. As mulheres casadas são mais propensas a iniciar e continuar a amamentação do que as mães solteiras, isto acontece, principalmente, pelo suporte físico e emocional ofertado com o compartilhamento de atividades domésticas (KORTSMIT et al., 2020).

Importante ressaltar que a forma como o homem se inclui durante o pré-natal deixa claro como ele irá se portar nos outros momentos do ciclo gravídico-puerperal. Esse envolvimento traz benefícios também no momento do parto, contribuindo com 
a sua participação ativa e no puerpério, momento em que a mulher vivencia as dificuldades de cuidar do recém-nascido (MAAS et al., 2012; FRANCISCO et al., 2015).

Existem outros fatores que influenciam a participação do companheiro nas consultas, como alta escolaridade materna, gravidez planejada, desejo materno de engravidar naquele período da vida, mulheres que iniciaram precocemente com o planejamento reprodutivo, gestantes que realizaram no mínimo seis consultas de pré-natal. Em contrapartida, a baixa adesão do homem vem diante da realização do acompanhamento da gestação em serviços públicos (BRITO et al., 2021).

Apesar dos benefícios, existem barreiras que dificultam 0 seu comparecimento às consultas de pré-natal, como a incompatibilidade de horário de funcionamento dos serviços de saúde com as atividades laborais. Por isso, é preciso que haja uma articulação que assegure ao homem o direito à parentalidade, assim como é garantida à mulher, sem que haja prejuízos no exercício da profissão e financeiro (CALDEIRA et al., 2017; LIMA et al., 2021; ONYEZE-JOE; GODIN, 2020; FIROUZAN et al., 2019; BRITO et al., 2021).

Segundo Onyeze-Joe e Godin (2020) os pais que trabalham na área rural têm mais probabilidade de comparecer a essas consultas, geralmente os agricultores ou proprietários de empresas local, quando comparados aos cônjuges que residem e exercem sua profissão em ambiente urbano. O comparecimento às consultas foi motivada pela curiosidade em saber da gravidez e pelo sentimento de felicidade de suas esposas quando participavam.

Somando-se a isto, algumas mulheres relatam sentir vergonha da presença do companheiro principalmente pelas mudanças ocasionadas pela gestação e faz com que ela se sinta insegura com relação à sua imagem. Por isso, os profissionais de saúde precisam estar atentos no reconhecimento de sentimentos negativos e identificação da relação da mulher com seu parceiro e como este encara a gestação e as mudanças ocasionadas por ela. É importante que haja a identificação desses fatores psicossociais, sejam eles negativos ou positivos, para que o processo seja vivenciado sem transtornos a fim de não interferir nas condutas adotadas (CALDEIRA et al., 2017). 
Além disso, existe uma barreira estrutural no próprio serviço de saúde que impossibilita o acompanhamento nas consultas e exames da mulher com restrições ou proibições da presença masculina nas clínicas de pré-natal e maternidades. Este fato pode ser evidenciado a partir do momento que a mulher não possui acesso a uma sala de PPP (pré-parto, parto e pós-parto) individual, de forma que garanta a privacidade, sem que haja o constrangimento para outras mulheres da presença do homem (FIROUZAN et al., 2019).

Apesar dos entraves encontrados, os profissionais de saúde da Atenção Primária à Saúde devem buscar estratégias de inclusão do companheiro para que ele usufrua dos inúmeros benefícios de sua participação, tanto para si quanto para a mãe e filho. É preciso que o incentivo seja tanto para as consultas de pré-natal quanto para demais atividades realizadas com gestantes (CALDEIRA et al., 2017).

A partir disso, o homem passará a compreender os processos biopsicossociais vivenciados pela mulher e contribuirá para uma vivência mais prazerosa e confiante a partir dos conhecimentos adquiridos durante as consultas, pois deste modo o parceiro se sentirá seguro para oferecer suporte adequado à mulher e a à criança (BRITO, et al., 2021; CALDEIRA et al., 2017).

Visando uma maior participação dos companheiros nas consultas de prénatal, estudos trazem estratégias utilizadas pelos profissionais para incentivar o comparecimento, como enviar mensagens de textos com conteúdo direcionado aos aspectos da gestação, convites enviados pela equipe de saúde para o homem para as consultas ou demais atividades a serem realizadas no serviço, dentre outros (BONIFÁCIO; SOUZA; VIEIRA, 2019; LIMA et al., 2021).

\section{CONCLUSÃO}

Diante do exposto, foi possível observar que vários são os benefícios que a participação ativa do parceiro traz para o ciclo gravídico. Por mais que culturalmente esta participação não seja estimulada, as políticas públicas querem garantir ainda mais que a paternidade seja exercida antes do nascimento da criança. 
Os benefícios variam desde ao aproveitamento da ida do homem ao serviço de saúde como oportunidade para realizar exames e consultas pertinentes à sua saúde como a formação de vínculo entre pai-mãe-bebê, encorajamento na realização de exames pela mulher, proporcionar uma participação ativa durante o trabalho de parto, assim como melhor cuidado com o recém-nascido e estímulo na amamentação.

Diversos estudos trazem quais são os fatores que incentivam esta participação, como por exemplo: a alta escolaridade materna, realização do pré-natal no serviço privado de saúde, seis ou mais consultas, satisfação e segurança da mulher com a presença do parceiro e incentivo dos profissionais.

Em contrapartida, existem os que interferem negativamente, como a incompatibilidade de horários do serviço de saúde e das atividades profissionais, impossibilitando o comparecimento do homem nas consultas e demais atividades propostas pelos profissionais, assim como fatores psicoemocionais da mulher, como vergonha do próprio corpo das modificações da gravidez e até aspectos estruturais dos próprios serviços de saúde que impossibilita a presença masculina dentro dos consultórios ou salas de parto.

Por isso, é preciso que a equipe da Atenção Primária à Saúde identifique essas barreiras precocemente para atuar de forma assertiva e eficaz a fim de que a mulher tenha todo o suporte necessário nas etapas da concepção, gestação, parto e puerpério. Vale salientar que os profissionais podem lançar de estratégias, como convites endereçados ao pai, para estimular essa participação, por exemplo.

Como limitação do estudo está a dificuldade em encontrar pesquisas que abordem o sucesso ou insucesso de estratégias que podem ser utilizadas para captar esses parceiros. Neste sentido, faz-se necessário a realização de mais estudos que possibilitem aos profissionais de saúde alternativas de incentivar o PréNatal do Parceiro dentro da Atenção Básica de Saúde, principalmente diante do achado de que uma das barreiras da não participação é o serviço público. 


\section{REFERÊNCIAS BIBLIOGRÁFICAS}

BALICA, L. O.; AGUIAR, R. S. Percepções paternas no acompanhamento do pré-natal. Revista de Atenção à Saúde (ISSN 2359-4330), v. 17, n. 61, 2019. Acesso em: 09 de out de 2021. Disponível em: http://seer.uscs.edu.br/index.php/revista_ciencias_saude/article/view/5934.

BENTO, A. V. Como fazer uma revisão da literatura: considerações teóricas e práticas. Rev JÁ, 7(65):42-44, 2012. Acesso em: 10 de set de 2021. Disponível em: http://www3.uma.pt/bento/Repositorio/Revisaodaliteratura.pdf.

BONIFÁCIO, L. P.; SOUZA, J. P.; VIEIRA, E. M. Adaptação de mensagens educativas para parceiros de gestantes para uso em tecnologias móveis em saúde (mHealth). InterfaceComunicação, Saúde, Educação, v. 23, 2019. Acesso em: 09 de dez de 2021. Disponível em: https://www.scielo.br/j/icse/a/cBPXmMgGmNxhxXDPwLZ8qZs/?format=html\&lang=pt.

BRASIL. Ministério da Saúde (BR). Portaria GM/MS no 3.562, de 12 de dezembro de 2021. Altera o Anexo XII da Portaria de Consolidação GM/MS no 2, de 28 de setembro de 2017, para dispor sobre a Política Nacional de Atenção Integral à Saúde do Homem (PNAISH). Ministério da Saúde, Secretaria de Atenção à Saúde- Brasília: Ministério da Saúde, 2021.

BRASIL. Secretaria de Atenção à Saúde. Departamento de Ações Programáticas Estratégicas. Guia do pré-natal do parceiro para profissionais de Saúde / Ministério da Saúde, Secretaria de Atenção à Saúde, Departamento de Ações Programáticas Estratégicas. - Brasília: Ministério da Saúde, 2018.

BRITO, J. G. E., et al. Participação do companheiro da gestante nas consultas de pré-natal: prevalência e fatores associados. Cogitare Enfermagem, v. 26, 2021. Acesso em: 19 de jan de 2022. Disponível em: https://www.scielo.br/j/cenf/a/qMhg65jGmBMcXzGdYDBqyrQ/abstract/?lang=pt.

CALDEIRA, L. Á., et al. A visão das gestantes acerca da participação do homem no processo gestacional. Revista de Enfermagem do Centro-Oeste Mineiro, v. 7, 2017. Acesso em: 13 de dez de 2021. Disponível em: http://seer.ufsj.edu.br/index.php/recom/article/view/1417.

CHIKALIPO, M. C.; CHIRWA, E. M.; MUULA, A. S. Acceptability of couple antenatal education: A qualitative study of expectant couples attending antenatal clinics in Blantyre, Malawi. Malawi Medical Journal, v. 30, n. 3, pág. 146-151, 2018. Acesso em: 09 de dez de 2021. Disponível em: https://www.ajol.info/index.php/mmj/article/view/180004.

COSTA, D. K. P., et al. Cuidados de enfermagem no pré-natal e segurança do paciente: revisão integrativa. Rev. enferm. UFPE on line, p. 4909-4919, 2016. Acesso em: 04 de nov de 2021. Disponível em: https://pesquisa.bvsalud.org/portal/resource/pt/biblio-1031744.

FERREIRA, I. S., et al. Percepções de gestantes sobre o papel do companheiro nas consultas de pré-natal. 2016. Acesso em: 11 de out de 2021. Disponível em: http://www.periodicos.ufc.br/index.php/rene/article/view/3444.

FIROUZAN, V., et al. Barriers to men's participation in perinatal care: a qualitative study in Iran. BMC pregnancy and childbirth, v. 19, n. 1, p. 1-9, 2019. Acesso em: 02 de jan de 2022. Disponível em: https://link.springer.com/article/10.1186/s12884-019-2201-2.

FRANCISCO, B. S., et al. Percepções dos pais sobre suas vivências como acompanhantes durante o parto e nascimento. Revista Mineira de Enfermagem, v. 19, n. 3, p. 567-583, 2015. Acesso em: 05 de dez de 2021. Disponível em: https://www.reme.org.br/artigo/detalhes/1024. 
GOMES, R. N. S.; FILHA, F. S. S. C.; PORTELA, N. L. C. Avaliação da influência do abandono da assistência pré-natal na mortalidade fetal e neonatal. RPCFO, v. 9, n. 2, p. 416-421, 2017. Acesso em: 16 de dez de 2021. Disponível em: http://seer.unirio.br/index.php/cuidadofundamental/article/view/5426.

KORTSMIT, K., et al. Paternal Involvement and Maternal Perinatal Behaviors: Pregnancy Risk Assessment Monitoring System, 2012-2015. Public Health Reports, v. 135, n. 2, p. 253-261, 2020. Acesso em: 02 de jan de 2022. Disponível em: https://journals.sagepub.com/doi/abs/10.1177/0033354920904066.

LIMA, K. S. V., et al. Father's participation in prenatal care and childbirth: contributions of nurses' interventions. Investigación y Educación en Enfermería, v. 39, n. 2, 2021. Acesso em: 15 de dez de 2021. Disponível em: https://revistas.udea.edu.co/index.php/iee/article/view/346570.

MAAS, A. J. B. M et al. "Expectant parents": study protocol of a longitudinal study concerning prenatal (risk) factors and postnatal infant development, parenting, and parent-infant relationships. BMC Gravidez e Parto, v. 12, n. 1, pág. 1-8, 2012. Acesso em: 05 de dez de 2021. Disponível em: https://bmcpregnancychildbirth.biomedcentral.com/articles/10.1186/14712393-12-46.

MENDES, S.; SANTOS, K. C. Pré-natal masculino: a importância da participação do pai nas consultas de pré-natal. Enciclopédia biosfera, v. 16, n. 29, 2019. Acesso em: 10 de out de 2021. Disponível em: https://conhecer.org.br/ojs/index.php/biosfera/article/view/331/314.

MOREIRA, M. C. N.; GOMES, R.; RIBEIRO, C. R. E agora o homem vem?! Estratégias de atenção à saúde dos homens. Cadernos de Saúde Pública, v. 32, p. e00060015, 2016. Acesso em: 05 de dez de 2021. Disponível em: https://www.scielosp.org/article/csp/2016.v32n4/e00060015/.

ONYEZE-JOE, C.; GODIN, I. Experiences, views and needs of first-time fathers in pregnancyrelated care: a qualitative study in south-east Nigeria. BMC pregnancy and childbirth, v. 20, n. 1, p. 1-11, 2020. Acesso em: 09 de jan de 2022. Disponível em: https://link.springer.com/article/10.1186/s12884-020-02889-w.

SANTOS, S C.; KREUTZ, C. M. O envolvimento do pai na gestação do primeiro filho. Pensando fam., Porto Alegre, v.18, n.2, p 62-76, dez. 2014. Acesso em: 09 de out de 2021. Disponível em http://pepsic.bvsalud.org/scielo.php?script=sci_arttext\&pid=S1679-

494X2014000200006\&lng=pt\&nrm=iso. 